\title{
Long-Range Attraction between Charge-Mosaic Surfaces across Water
}

\author{
Susan Perkin, ${ }^{1}$ Nir Kampf, ${ }^{2}$ and Jacob Klein ${ }^{1,2}$ \\ ${ }^{1}$ Physical and Theoretical Chemistry Laboratory, Oxford University, Oxford OX1 3QZ, United Kingdom \\ ${ }^{2}$ Department of Materials and Interfaces, Weizmann Institute, Rehovot 76100, Israel
}

(Received 11 May 2005; published 23 January 2006)

\begin{abstract}
We have measured directly the forces across water between hydrophilic surfaces covered with a random mosaic of positive and negative charged domains. We find a strong, long-ranged attraction between them at a surface separation comparable with the charge domain size (many tens of nanometers). This attraction persists at higher salt concentration, but its range then becomes comparable to the Debye screening length. We attribute the attraction to correlation between negative and positive regions on opposing surfaces, facilitated by the lateral mobility of the charge patches on the surfaces.
\end{abstract}

DOI: 10.1103/PhysRevLett.96.038301

The electrostatic double-layer interaction between charged surfaces across water, together with the ubiquitous van der Waals (vdW) forces, are the major effects controlling interactions between hydrophilic surfaces in aqueous media [1-4]. Direct measurements have revealed the magnitude and range of such forces [3]. In real life, however, uniformly charged surfaces are the exception rather than the rule, particularly in a biological context. Here we demonstrate that two interacting hydrophilic surfaces, each covered by a mosaic of positively and negatively charged domains, experience a strong attraction for each other across water (no added salt) at a range of ca. 50 nanometers, comparable to the size of the domains. At higher salt concentration the range of the attraction becomes smaller than that of the domains and comparable to the Debye screening length, a crossover consistent with theoretical predictions.

Surfaces bearing a mosaic of positive and negative charge patches were prepared by coating freshly cleaved, molecularly smooth mica surfaces with a smooth, uniform monolayer of a cationic surfactant [5] and then immersing in surfactant-free purified water or in aqueous $0.01 \mathrm{M} \mathrm{NaCl}$ [6]. With increasing immersion time the initially hydrophobic surfaces became progressively more hydrophilic, as revealed by contact angle measurements [Fig. 1(a)]. The corresponding structure of the surfactant monolayer is monitored using a tapping-mode atomic force microscope (AFM), as shown in Fig. 1. Immediately after its selfassembly from solution and for a short period following immersion in water, the monolayer coating the surface was uniform and smooth with thickness ca. $1.5 \pm 0.2 \mathrm{~nm}$, Fig. 1(b). With progressive immersion in water or salt solution the initially hydrophobic coating breaks up into a series of irregular patches or rafts of uniform thickness roughly double that of the monolayer, as shown in Figs. 1(c) and 1(d). These have lateral dimensions in the range ca. 50-400 nm, and are surrounded by a continuous smooth substrate. Analysis of the AFM micrographs reveals that close to $50 \%$ of the surface may be covered with such rafts of double the monolayer thickness, indicating that the remaining (ca. 50\%) substrate area consists of
PACS numbers: 82.70.Uv, 68.08.De, 68.43.Hn, 68.37.Ps

essentially bare mica. Since the cationic surfactant bilayers expose a positively charged head group at the water interface, and since mica is known to be negatively charged in water, this implies that the initially smooth and uniform monolayer breaks up into a mosaic of positively charged bilayer rafts surrounded by bare, negatively charged mica. This is shown in the cartoons in Fig. 1, and is consistent with the corresponding increased wettability of the surfaces [Fig. 1(a)] following immersion in water or in aqueous salt solution [7].

Force-distance profiles $F(D)$ between such surfaces were measured directly as a function of their separation $D$ in a surface force balance (SFB) as earlier described [8], across water or across aqueous $0.01 \mathrm{M} \mathrm{NaCl}$, as shown in Figs. 2(a) and 2(b), respectively, and summarized in Table I. The normalized profiles $(F / R)$ versus $D$ (where $R \approx 10^{-2} \mathrm{~m}$ is the mean radius of curvature of the mica sheets) were first measured as a control between the bare mica surfaces prior to their coating by the surfactant. In water [asterisks, Fig. 2(a)] these reveal the expected longranged repulsion due to double-layer interactions, followed by a jump-due to an Euler-like spring instability driven by vdW attraction - from short range (ca. $4 \mathrm{~nm}$, Table I) into adhesive contact [3,9]. Following this, the lensmounted mica surfaces were removed and coated as described [5], remounted in the SFB, and forces between them were measured again at increasing immersion times. We consider first interactions across water with no added salt, Fig. 2(a) [10]. The interactions at short times between the initially smooth, hydrophobic layers [Fig. 2(a), open symbols] are, as expected, [11] long-ranged and strongly attractive, jumping from separation $D_{j}$ into contact at separation $D_{0}$.

Forces were subsequently measured following progressively longer immersion in water, as shown by the filled symbols in Fig. 2(a) and in part A of Table I. The remarkable feature of these profiles is that, despite the progressive breakup of the originally smooth monolayers into a hydrophilic mosaic of positive and negative charges [as in Fig. 1(c)], their interactions remain attractive and long ranged, indeed even slightly longer ranged than prior to 

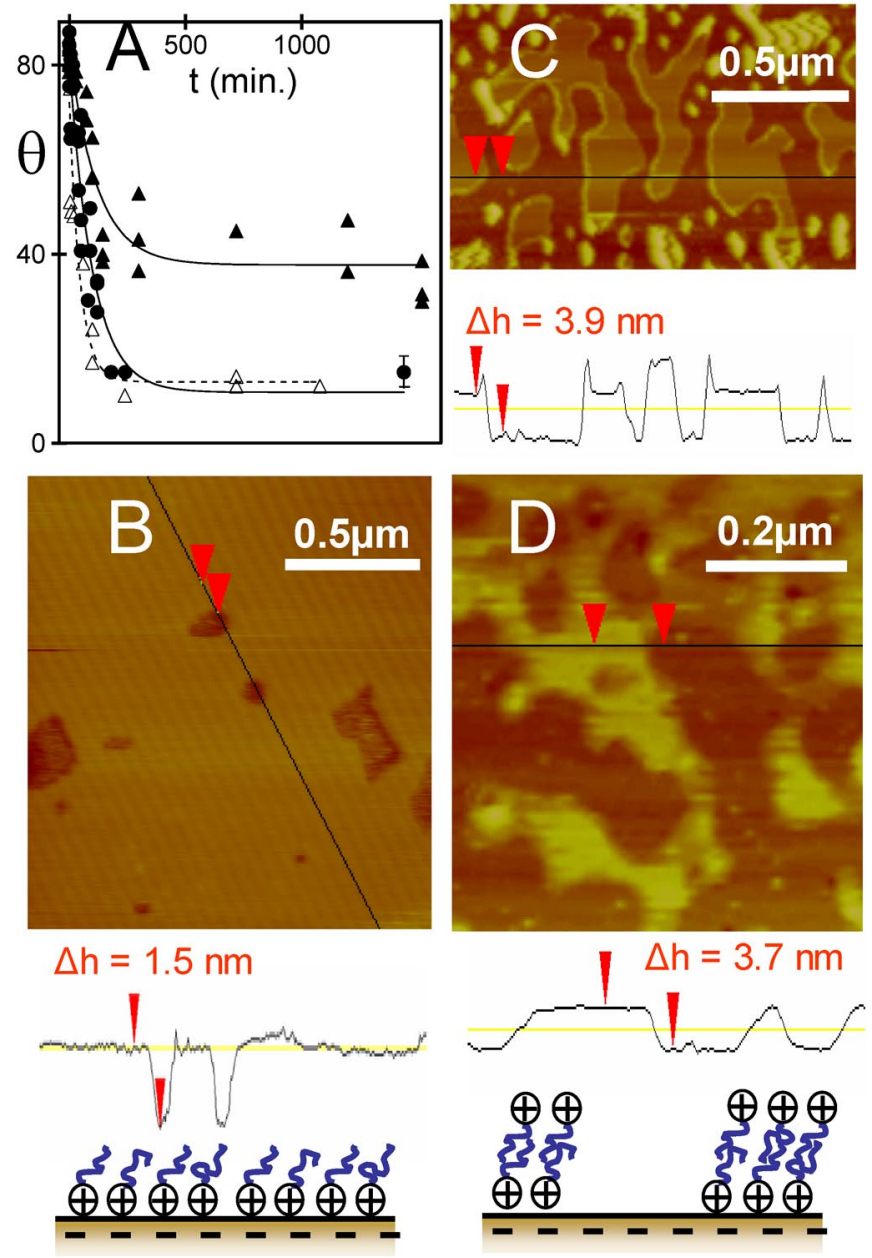

FIG. 1 (color online). (a) Advancing contact angle $\Theta$ of a water droplet on the mica surface: $\boldsymbol{\Delta}$-immersion in water; $\triangle$-immersion in aqueous $0.01 \mathrm{M} \mathrm{NaCl}$; - immersion in water for mica incubated for $30 \mathrm{sec}$ [rather than $2 \mathrm{~h}$ [5]] in the surfactant solution. Mica sheets were never reimmersed: each data point corresponds to a new mica sheet [7]. (b) Tapping-mode AFM images (fluid cell, Nanoscope III, Digital Instruments) of a coated mica surface under water [30 sec incubation in surfactant solution [5] ], 5 min after coverage of the surface by the water. Dark areas are holes, $1.5 \pm$ $0.2 \mathrm{~nm}$ deep, in the surfactant monolayer. (c) As (b), but following $30 \mathrm{~min}$ immersion in water. (d) Coated mica following 11 min immersion in $0.01 M \mathrm{NaCl}$, showing similar bilayer islands to (c), with cross section indicated below image. Bottom cartoon: the initially smooth monolayer [left, as in (b)] rearranges on immersion in water or $0.01 \mathrm{M} \mathrm{NaCl}$ to patches of bilayer [right, as in (c), (d)] which bear a net positive charge, surrounded by the negatively charged bare mica.

the breakup. This is emphasized also in Table I which summarizes the separations $D_{j}$ from which jumps-in to contact occur. We note that the contact separation $D_{0}$ to which the surfaces jump under the long-ranged attraction is correlated with the state of the surfactant layer (Table I). $D_{0}$ values for jumps-in after short immersion of the surfaces in water, when the monolayers are still uniform and smooth, correspond to some twice the monolayer thick-

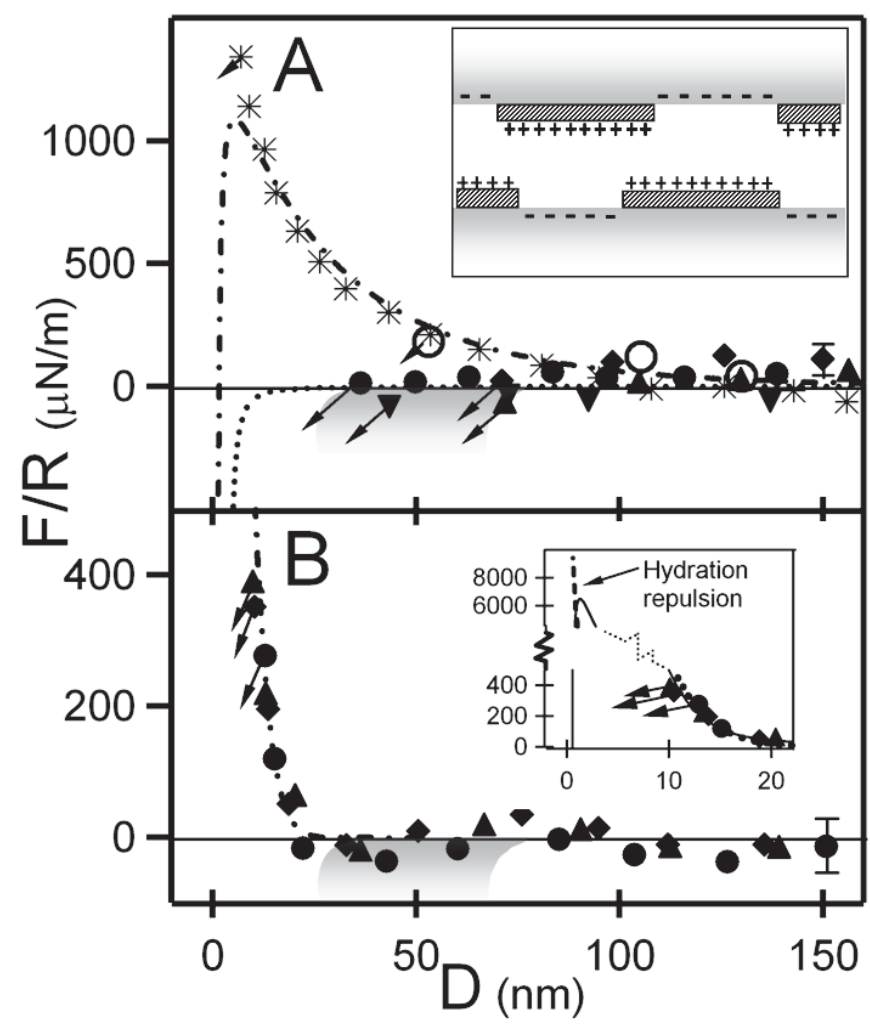

FIG. 2. SFB-measured force-distance profiles $F(D) / R$ vs $D$ between curved mica surfaces (mean radius of curvature $R$ ) a closest distance $D$ apart as described previously [8,9]. (a) Forces between bare $(*)$ and coated (all other symbols) [5] mica surfaces across water [6], fitted by a DLVO model [[1,2], dotdashed curves], using parameters of surface potential and effective ion concentration within the range of literature reports [24,27]. $\bigcirc$ : profiles taken within $95 \mathrm{~min}$ of adding water to the cell [surfaces hydrophobic, Fig. 1(a)]. Filled symbols are recorded between 2 and $17 \mathrm{~h}$ after adding water [surfaces hydrophilic, Fig. 1(a)]. The dotted curve is the predicted vdW interaction between the surfaces according to Ref. [13]. The cartoon inset shows the negatively charged mica surface regions on one surface largely facing the positively charged bilayer rafts on the other. (b) As (a) but in aqueous $0.01 \mathrm{M} \mathrm{NaCl}$. The shaded band shows the range of attractions measured in salt-free water from Fig. 2(a), while the data $(\boldsymbol{\bullet}, \boldsymbol{\Delta}, \boldsymbol{\diamond})$ are for immersion periods of 40-90 min in the salt solution. The curves are the calculated DLVO double-layer profile for this salt concentration (offset by twice the thickness of a surfactant bilayer) and a surface potential $40 \mathrm{mV}$, indicating uncompensated charge on the mica surfaces. Inset: profiles in the vicinity of the jump on an expanded scale, showing the DLVO prediction up to the peak where vdW attraction overcomes the double-layer repulsion. The broken curve in the inset indicates the hydration repulsion observed between charged bare mica surfaces across $\mathrm{NaCl}$ solutions of similar concentrations [25,28].

ness, as expected for monolayer-monolayer contact. At later times, corresponding to the development of the raftlike bilayer structure as typified in Figs. 1(c) or 1(d), the $D_{0}$ values increase to some 4 times the monolayer thickness, as expected for bilayer-bilayer contact. 
TABLE I. Summary of surface separations $D_{j}$ from which mica surfaces jump into adhesive contact at $D_{0}$. A and $\mathrm{B}$ are taken from surface force profiles such as in Fig. 2(a) and 2(b), respectively.

\begin{tabular}{lcccc}
\hline \hline Surfaces & $\begin{array}{c}\text { Immersion } \\
\text { medium }\end{array}$ & $\begin{array}{c}\text { Immersion } \\
\text { time }\end{array}$ & $D_{j}(\mathrm{~nm})$ & $D_{0}(\mathrm{~nm})$ \\
\hline $\mathrm{A}$ & & & & \\
(Bare mica & Water & & $4.1 \pm 2.5$ & $0.0 \pm 0.9)$ \\
Coated mica & Water & $20-95 \mathrm{~min}$ & $32 \pm 21$ & $2.5 \pm 0.4$ \\
Coated mica & Water & $2-5 \mathrm{~h}$ & $40 \pm 19$ & $5.7 \pm 1.6$ \\
Coated mica & Water & $14-17 \mathrm{~h}$ & $50 \pm 20$ & $6.5 \pm 1.2$ \\
B & & & $3.6 \pm 2.0$ & $0.0 \pm 0.8)$ \\
(Bare mica & Water & & $4.1 \pm 1.7$ & $4.0 \pm 0.3$ \\
Coated mica & $0.01 M \mathrm{NaCl}$ & $40-90 \mathrm{~min}$ & $11.1 \pm$ & \\
\hline \hline
\end{tabular}

The attractive forces near the point where the surfaces jump spontaneously into contact at surface separation, $D=D_{j}$, may be evaluated from the condition at a jump instability $(\partial F / \partial D)_{D=D_{j}}=K$, the normal spring constant. We approximate $(\partial F / \partial D)$ by $\delta F / \delta D \approx K$, where the mean value of $|\delta D|$ is half the approaching step size $\Delta D_{0}$ (Fig. 2) at $D=D_{j}$. Then, since the surfaces jump together from a point between $D_{j}$ and $\left(D_{j}-\delta D\right), \delta F=$ $K .|\delta D|$ is a lower limit on the magnitude of the attractive force $F\left(D_{j}-\delta D\right)$ when the separation between the surfaces is $D=\left(D_{j}-\delta D\right)$. Putting $K=160 \mathrm{~N} / \mathrm{m}$ and $\delta D=\left(\Delta D_{0} / 2\right)=$ ca. $6 \mathrm{~nm}$, we find $F\left(D_{j}-\delta D\right) \approx$ $-10^{-6} \mathrm{~N}$. This is some 200 times larger than the expected nonretarded van der Waals attraction $F_{\mathrm{vdW}}\left(D_{j}-\delta D\right)=$ $-\left(A R / 6\left(D_{j}-\delta D\right)^{2}\right) \approx-5 \times 10^{-9} \mathrm{~N}$ [where $A \approx 1.5 \times$ $10^{-20} \mathrm{~J}$ is the Hamaker constant for hydrocarbon-coated mica surfaces across water [3]]. In practice the van der Waals forces at these values of $D_{j}$ will be retarded [3], and therefore much lower still, so our measured attraction at $D_{j}$ between the charge-moasic surfaces is at least 3 or more orders of magnitude larger than the expected $\mathrm{vdW}$ forces at that separation.

What is the origin of this behavior, where hydrophilic surfaces covered by domains of positive and negative charges experience a strong, long-ranged attraction for each other across water? We attribute the attractive forces to a correlation between oppositely charged domains on the interacting surfaces as they face each other. Such a correlation, we believe, may develop as the charged bilayer rafts on each surface adjust their position when they approach [12], driven by the lower free energy associated with such a configuration, so that positively charged regions on one surface face mostly negatively charged bare mica surface regions on the other [cartoon inset to Fig. 2(a)]. We note that strong attraction across aqueous media has long been known between hydrophobic surfaces [as for the open symbols in Fig. 2(a)], often hydrophobized with self-assembled layers of amphiphilic surfactant molecules in a solution of the surfactant [11]. Explanations of the hydrophobic attraction have ranged from an adsorption-instability model [13] of surface attractions within surfactant solutions [14,15], to conjectures that it may be associated with electrostatic attraction $[16,17]$ between surfaces with mixed charges [15,18-22]. Our mosaic-charge surfaces are clearly hydrophilic rather than hydrophobic and so differ conceptually from the earlier work [11]: the present study unambiguously demonstrates the strong, long-ranged attraction that is possible between surfaces covered by domains of mixed charge.

Models of interactions between mixed charge domains in salt solutions indicate an attraction whose range depends on both domain size and the Debye screening length [19$21,23]$. A configuration quite similar to ours was treated in Ref. [23], evaluating the interaction between two overallneutral surfaces bearing positively and negatively charged domains of radius $P$ (with positive domains on one surface facing negative domains on the other). The resulting predicted long-ranged attraction across water has a magnitude at large surface separations $D$ decreasing exponentially as $e^{-D / \delta}$, with a decay length $\delta \approx\left[\kappa^{2}+\left(C / P^{2}\right)\right]^{-1 / 2}$, where $\kappa^{-1}$ is the Debye screening length across the aqueous medium between the surfaces, and $C$ a constant of order unity (depending on the precise shape of the charged domains). The range of the attraction is then determined largely by the shorter of $\kappa^{-1}$ or $P$. In our experiments the charge domains [Figs. 1(c) and 1(d)] vary in size and shape, so that $P$ is not precisely defined, but it is noteworthy that the range $50 \pm 20 \mathrm{~nm}$ (Table I) of the attraction between the charge-mosaic-covered surfaces across water with no added salt, Fig. 2(a), is comparable with the lower size range $2 P \approx 50-100 \mathrm{~nm}$ of the positive charge domains, Figs. 1(c) and 1(d) . The magnitude of the attraction at the jump positions $D=D_{j}$ [Fig. 2(a)] is also comparable with that predicted by this model [23].

To get further insight we measured forces between our charge-mosaic surfaces in $0.01 \mathrm{M}$ aqueous $\mathrm{NaCl}$ solution, as shown in Fig. 2(b). The surface forces, after immersion times long enough to hydrophilize the surfaces [Figs. 1(a) and 1(d)], are weakly repulsive at larger separations due to a small net residual surface charge, but as they approach, a strong attraction causes the surfaces to jump into contact. Such attraction is very different to the monotonic hydration repulsion (due to trapped hydrated counterions) observed between bare charged mica surfaces across $0.01 M \mathrm{NaCl}$ $[25,26]$. Its range and magnitude, though much stronger and longer ranged than expected from $\mathrm{vdW}$ forces [inset in Fig. 2(b)], is strikingly smaller than for the case of the saltfree water [Fig. 2(a), summarized as gray-shaded zone in Fig. 2(b)]. The jump distances ( $D_{j}$ to $D_{o}$, Table I), characteristic of the range of the attractive interaction, are of order 6-7 nm, compared with $\kappa^{-1} \approx 3 \mathrm{~nm}$ in $0.01 M \mathrm{NaCl}$ and compared with the attraction range of $50 \pm 20 \mathrm{~nm}$ in the salt-free water and with the (lower) values of $P \approx$ 25-50 nm [Figs. 1(c) and 1(d)]. This crossover of the range of the attraction, from a value similar to the charge domain size $P$ in salt-free water, to much smaller values compa- 
rable with $\kappa^{-1}$ at the higher salt concentration, is in line with expectations $[19,23]$.

In summary, we have shown that two (hydrophilic) surfaces covered by domains of positive and negative charge may attract each other strongly across water: the magnitude of this attraction is at least 3 or more orders of magnitude greater than the van der Waals attraction at surface separations of some tens of $\mathrm{nm}$, a range comparable with the size of the (smaller) domains. On increasing the salt concentration the range of the strong attraction is much reduced, becoming comparable with the correspondingly reduced Debye screening length. We believe this attraction, currently being explored more systematically, is due to a correlation between oppositely charged regions on the interacting surfaces which arises - as the surfaces approach each other - from lateral rearrangement of the mobile charged regions. These results have basic implications for the forces that act across water between surfaces bearing regions of mixed charge, as often found at biological interfaces such as those of cells and of proteins as well as on colloidal particles.

We are grateful to Sidney Cohen for his help with the AFM imaging, and for useful discussions and correspondence with Håkan Wennerström, Rob Atkin, Colin Bain, Phil Pincus, Sam Safran, and Richard Zare. We thank the EPSRC (UK) and the Israel Science Foundation for financial support.

Note added in proof. - An attractive interaction between charge mosaic surfaces, similar to that published earlier by S. Perkin et al. [11], was reported by E. Meyer et al. [29].

[1] B. V. Derjaguin and L. Landau, Acta Physicochim URSS 14, 633 (1941).

[2] E. J. W. Verwey and J. T. G. Overbeek, Theory of the Stability of Lyophobic Colloids (Elsevier, New York, 1948).

[3] J.N. Israelachvili, Intermolecular and Surface Forces (Academic, New York, 1992).

[4] S. A. Safran, Statistical Thermodynamics of Surfaces, Interfaces and Membranes (Addison-Wesley, Reading, MA, 1994).

[5] Freshly cleaved mica surfaces were incubated for $2 \mathrm{~h}$ in a $0.1 \% \mathrm{w} / \mathrm{w}$ solution of cetyltrimethylammonium bromide $\left(\mathrm{CH}_{3}\left(\mathrm{CH}_{2}\right)_{15} \mathrm{~N}^{+}\left(\mathrm{CH}_{3}\right)_{3} \mathrm{Br}^{-}\right)$[CTAB; Fluka Puriss, $>99 \%$, in water, to emerge smooth, dry, and hydrophobic [Figs. 1(a) and 1(b)].

[6] Tap water was passed through a $\mathrm{RiOs}^{\mathrm{TM}}$ reverse-osmosis stage followed by purification in a Milli- $Q$ Gradient $^{\mathrm{TM}}$ system: it had a resistivity $\geq 18.2 \mathrm{M} \Omega$, and total organic content $\leq 4 \mathrm{ppb}$. The $\mathrm{NaCl}$ used (Aldrich, 99.999\%) was UV irradiated for $60 \mathrm{~min}$ before dissolution in the water.

[7] Withdrawal from water of the mica sheets removes the upper monolayer of the bilayer rafts, exposing the hydrophobic alkyl tails of the lower layer, resulting in finite contact angles at long immersion times [Fig. 1(a)]. The immersed surfaces fully covered by charged patches are expected to be completely wetted by water.

[8] J. Klein and E. Kumacheva, J. Chem. Phys. 108, 6996 (1998).
[9] U. Raviv, P. Laurat, and J. Klein, Nature (London) 413, 51 (2001).

[10] Interactions between surfaces where monolayers were formed following $30 \mathrm{sec}$ incubation were qualitatively similar to those shown in Fig. 2(a), though of somewhat shorter range [S. Perkin, N. Kampf, and J. Klein, J. Phys. Chem. B 109, 3832 (2005)].

[11] H. K. Christenson and P. M. Claesson, Adv. Colloid Interface Sci. 91, 391 (2001).

[12] By evaluating the lateral attraction between two oppositely charged domains (effectively dipole domains) together with the lateral mobility of each domain, we estimate (to be published) a lateral domain velocity $v_{1} \approx$ $A(\sigma / e)\left(D_{1} / k_{B} T\right) f_{1}(\theta, D)$, where $A$ is the domain area, $\sigma$ its net charge, $e$ the electron charge, $D_{1}$ the lateral diffusion coefficient of a surfactant molecule on the substrate, and $f_{1}=\mid\left[\mu^{2} \cos ^{2} \theta /\left(4 \pi \varepsilon \varepsilon_{0} D^{4}\right)\right]\left(-15 \cos ^{4} \theta \sin \theta+\right.$ $\left.3 \cos ^{2} \theta \sin \theta\right) \mid$ is the lateral attraction between two dipoles each of moment $\mu$, where $\theta$ is the angle subtended between the dipoles and the surface normal. For typical values of the present configuration we find, for patches of extent $50 \mathrm{~nm}$ at surface separation $D=D_{j} \approx 50 \mathrm{~nm}$ and $\theta=450^{\circ}, v_{l} \approx O(10) \mathrm{nm} / \mathrm{sec}$, which is ample for domain correlation to occur over the jump into contact.

[13] R. Podgornik and V. A. Parsegian, J. Phys. Chem. 99, 9491 (1995).

[14] J. L. Parker, V. V. Yaminsky, and P. M. Claesson, J. Phys. Chem. 97, 7706 (1993).

[15] P. Kekicheff and O. Spalla, Phys. Rev. Lett. 75, 1851 (1995).

[16] V.A. Parsegian and D. Gingell, Biophys. J. 12, 1192 (1972).

[17] S. A. Safran, Europhys. Lett. 69, 826 (2005).

[18] R. Podgornik, J. Phys. Chem. 91, 5840 (1989).

[19] S. J. Miklavic et al., J. Phys. Chem. 98, 9022 (1994).

[20] J. Forsman, B. Jonsson, and T. Akesson, J. Phys. Chem. B 102, 5082 (1998).

[21] Y.-H. Tsao, D.F. Evans, and H. Wennerstrom, Science 262, 547 (1993).

[22] Note, however, that the attraction between opposing domains of mixed charge predicted in Refs. [19,20] is within the parameters accessible to these studies-comparable to van der Waals attraction, and very much weaker than that measured in our study at separations corresponding to the onset of attraction, Fig. 2(a).

[23] D. Fennel Evans and Hakan Wennerstrom, The Colloidal Domain (Wiley, New York, 1999), 2nd ed., section 5.6; see also Ref. [19].

[24] The screening length $\kappa^{-1}$ for interactions between bare mica across purified water with no added salt has been measured in the range up to $120 \pm 20 \mathrm{~nm}$ [e.g., U. Raviv et al., Langmuir 20, 5322 (2004)], corresponding to an effective $1: 1$ salt concentration $6 \times 10^{-6} \mathrm{M}$, though smaller values have also been measured, as in Fig. 2(a).

[25] U. Raviv and J. Klein, Science 297, 1540 (2002).

[26] R. M. Pashley, J. Colloid Interface Sci. 80, 153 (1981).

[27] U. Raviv, P. Laurat, and J. Klein, J. Chem. Phys. 116, 5167 (2002).

[28] R. M. Pashley, J. Colloid Interface Sci. 83, 531 (1981).

[29] E. Meyer et al., Proc. Natl. Acad. Sci. U.S.A. 102, 6839 (2005). 